05

\title{
Особенности процессов накопления и транспорта электретных зарядов в мелкоразмерных разупорядоченных структурах под действием внутреннего напряжения
}

\author{
(C) А.Б. Танаев, Л.А. Щербаченко, Я.В. Безрукова, Ш.Б. Цыдыпов, \\ М.Ю. Бузунова, С.С. Барышников, Л.И. Ежова, Д.С. Барышников
}

Иркутский государственный университет,

664003 Иркутск, Россия

ฯ e-mail: irk.sherbachenko@mail.ru

(Поступило в Редакцию 16 апреля 2016 г.)

\begin{abstract}
Проведено исследование электретных свойств и структурных особенностей в мелкодисперсных гетерогенных системах на примере механоактивированных зерен природных углей, погруженных в полярную водную матрицу. Наблюдаемая аккумуляция токов при отсутствии внешнего напряжения свидетельствует о формировании разности потенциалов внутреннего электрического поля в изучаемых объектах. Показано, что на межфазных границах в исследуемой системе образуется слой водной фазы с жесткой фиксацией молекул воды, способный приобретать функции потенциального барьера, затрудняющего переход этого слоя как свободными молекулами воды, так и молекулами воды, ориентированными внутренним электрическим полем системы.
\end{abstract}

DOI: 10.21883/JTF.2017.03.44243.1388

\section{Введение}

В последнее время заметное внимание уделяется исследованию мелкодисперсных механоактивированных систем с развитой удельной поверхностью. Интерес к таким структурам вызван регистрацией новых физических свойств, связанных с аккумуляцией электрического тока в таких объектах при отсутствии внешнего напряжения, а также с формированием упорядоченной ориентации молекул полярных водных пленок, находящихся в контакте с электрически активной поверхностью механоактивированных частиц твердой фазы. В таких неравновесных системах возрастает эффективность адсорбционных процессов, вызванная наличием заряженных дефектов на поверхности мелкоразмерных зерен природных углей, приводящих к образованию водных пленок обволакивающих мелкоразмерные частицы твердой компоненты. Наличие и количество воды в межслоевом пространстве не влияет на структуру кристаллической решетки углей, но, тем не менее, расстояние молекул воды в межслоевом пространстве по характеру взаимодействия с ближайшим окружением может быть различным.

На межфазных границах в рассматриваемой гетерогенной системе возникает кулоновское взаимодействие зарядов, расположенных на электрически активной поверхности минеральных частиц углей с полярными молекулами и ионами водной пленки. При наличии многочисленных границ раздела в исследуемых объектах суммарный эффект межфазных взаимодействий становится более сильным и является определяющим для процесса генерации внутренних электрических полей [1]. Источниками таких полей являются заряженные дефекты, локализованные на различных энергетических уровнях как поверхности, так и в объеме твердой компоненты, а также свободные ионы и полярные молекулы водной фазы [2].

Внутреннее электрическое поле является катализатором процессов диссоциации водной компоненты, что способствует увеличению в ней концентрации свободных зарядов. Смещение заряженных частиц в водной пленке под действием устойчивого внутреннего поля в новые энергетически более выгодные позиции их закрепления обеспечивает миграцию ионов к активной поверхности твердой фазы, а также их накопление в объеме исследуемой электрически активной системы, что свидетельствует о проявлении в ней естественных электретных свойств. Цель настоящей работы заключалась в изучении структурных и электретных свойств, а также выяснение особенностей генерации внутренней электрической энергии в образцах мелкодисперсных природных углей.

В работе использовался метод термостимулированной спектроскопии (ТСС), позволяющий исследовать процессы релаксации и переноса электретных свободных зарядов, проявляющихся при нагревании изучаемых структур. Использование этого метода позволяет получить информацию о состоянии структурных особенностей электрически активных систем, а также выяснить механизм межфазного взаимодействия их компонентов [3]. Исследование динамики процесса реализации термостимулированных токов при нагревании изучаемых объектов позволяет установить природу доминирующих в них релаксаторов и выявить условия разрушения дипольной поляризации [4]. 


\section{Методика эксперимента}

Экспериментальные образцы изготавливались посредством механоактивации природных углей Черемховского бассейна в установке Pulverisette 5, с помощью которой были получены дисперсные системы с размерами частиц в интервале от 31 до $105 \mu \mathrm{m}$. Гранулометрический состав изучаемых структур более детально определялся с помощью лазерного анализатора частиц MicroSizer 201. Величина адсорбции исследуемых образцов при комнатной температуре составляла $\varphi=2.1 \%$.

Для получения заданных величин адсорбции образцы выдерживались во влажной среде эксикатора с насыщенным раствором кальцинированной соды $\mathrm{Na}_{2} \mathrm{CO}_{3}$. Подготовленные объекты являлись двукомпонентными гетерогенными системами, в которых полярная водная фаза контактировала с электрически активной поверхностью мелкоразмерных минеральных частиц природных углей.

Изучаемые образцы фиксированной массы помещались в специально сконструированную измерительную ячейку, представляющую собой плоский конденсатор с алюминиевыми электродами диаметром $1.5 \mathrm{~cm}$. На электроды измерительной ячейки, где находился изучаемый образец, внешнее напряжение не подавалось. Исследуемые объекты подвергались нагреванию. В таких условиях экспериментально наблюдаемые спектры термостимулированных токов исследуемых структур зависели только от распределения собственных внутренних электрических полей, создаваемых локализованными носителями термоэлектретного заряда.

Регистрация термостимулированных токов производилась в режиме короткозамкнутого образца при его линейном нагреве с постоянной скоростью $\beta$, не превышающей $1 \mathrm{deg} / \mathrm{min}$ в исследуемом интервале температур от $20^{\circ} \mathrm{C}$ до $620^{\circ} \mathrm{C}$ с помощью чувствительного вольтметра-электрометра В7-49 (погрешность измерения тока $10^{-15} \mathrm{~A}$ ). Низкая скорость нагрева обеспечивала отсутствие градиентов температуры в образце, что являлось необходимым условием получения информативных и достоверных термостимулированных токовых спектров. Полученные экспериментальные результаты записывались в память персонального компьютера, обрабатывались стандартными статистическими методами и были представлены в виде временнб́х диаграмм термостимулированных токов и температуры образца. Наблюдение динамики термостимулированных токов позволяло получить информацию о природе электретного состояния и электрофизических характеристиках исследуемых объектов [5].

По результатам наблюдений рассчитывались основные термоэлектретные параметры: энергия активации носителей заряда вычислялась по формуле $U_{n} \sim 27 \mathrm{kT}$, где $k$ - постоянная Больцмана, $T-$ абсолютная температура, соответствующая положению максимума термотока, время релаксации структурных элементов $\tau$ определялось по максимуму термостимулированного тока при температуре $T_{\max }$ :

$$
\tau=\frac{k}{\beta U_{n}}\left[T_{\max }\right]^{2},
$$

где $\beta-$ скорость нагрева системы.

\section{Результаты эксперимента и обсуждение}

На рис. 1 представлены термограммы для образцов мелкоразмерных природных углей, погруженных в водную полярную матрицу. В температурном интервале от 20 до $620^{\circ} \mathrm{C}$ наблюдается пять доминирующих термомаксимумов тока, динамика которых в рассматриваемых температурных интервалах представлена локальными резонансными максимумами тока, что свидетельствует о неоднородности исследуемой системы. Формирование максимумов в спектрах термотоков при нагревании образцов вызвано образованием структурных поляризационных заряженных дефектов, которые до начала процесса термической активации находились в потенциальных ловушках изучаемых объектов. Каждый максимум тока в спектрах термостимулированных токов (ТСТ) соответствует электретному заряду определенного типа. Интенсивная релаксация термостимулированных токов при отсутствии внешнего напряжения подтверждает наличие внутренней разности потенциалов в рассматриваемых структурах, способствующей направленному переносу свободных носителей электретных зарядов [6].

Изучаемая неравновесная мелкодисперсная система природных механоактивированных зерен углей характеризуется пространственно неоднородным распределением электретных зарядов, способных в определенном интервале температур, приобретая дополнительную энергию при повышении температуры, проявляться и влиять на интенсивность разрядных термостимулированных токов. Релаксация разрядного тока сопровождается появлением максимумов термотока различной природы, что характеризует наличие сложного порядка в расположении атомов данной системы, а также локализации зарядов на различных дефектах.

В спектрах ТСТ прослеживаются две температурные области. В низкотемпературной области от 20 до $120^{\circ} \mathrm{C}$ для всех трех исследуемых структур преобладающими источниками направленно движущихся электретных зарядов являются процессы диссоциации полярных водных молекул. Выявлено, что наличие доминирующих максимумов в низкотемпературной области связывается с особенностями структуры водной фазы и накоплением межфазных свободных электретных зарядов под действием внутреннего электрического поля [7].

Пик термотока в окрестности температуры $27^{\circ} \mathrm{C}$, по всей вероятности, вызван термически стимулированным движением свободных гетерозарядов, накопленных вблизи электроконтактных границ раздела разнородных фаз под действием внутреннего поля системы. Согласно 

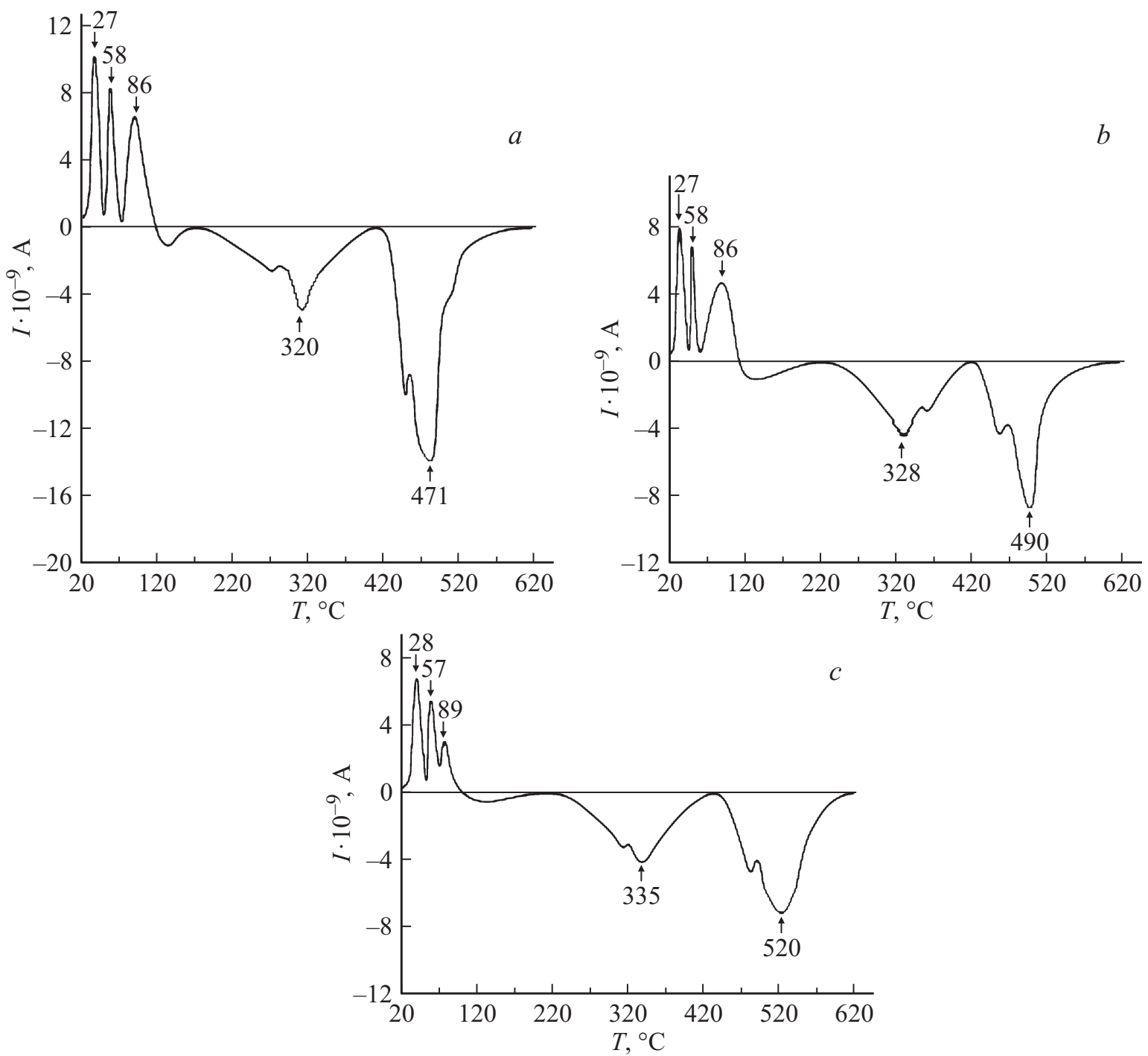

Рис. 1. Спектры термостимулированных токов механоактивированных мелкодисперсных образцов природных углей при адсорбции водной компоненты $\varphi=2.1 \%$ : $a$ - спектры образца 1 (дисперсность $32-42 \mu \mathrm{m}$ ); $b$ - спектры образца 2 (дисперсность 54-69 $\mu \mathrm{m}) ; c-$ спектры образца 3 (дисперсность 82-105 $\mu \mathrm{m})$.

теории Пула-Френкеля, диссоциация молекул жидкости при низких температурах осуществляется преимущественно под действием внутреннего электрического поля, поскольку энергии теплового движения молекул для их ионизации недостаточно [8].

Наибольшая интенсивность максимума релаксационного тока $I_{\max 1}=10.6 \cdot 10^{-9} \mathrm{~A}$ при температуре $27^{\circ} \mathrm{C}$ свидетельствует о значительной электрической активности изучаемого образца 1 и увеличении концентрации накопленных свободных зарядов на межфазных границах изучаемого объекта.

Интенсивный пик термотока для более электрически активного образца $1 I_{\max 1}=8.2 \cdot 10^{-9} \mathrm{~A}$ при $58^{\circ} \mathrm{C}$, по всей вероятности, свидетельствует о структурном переходе связанной воды в свободное состояние, что сопровождается приобретением молекулами полярной воды дополнительных степеней свободы за счет уве- личения энергии тепловых колебаний с ростом температуры системы [9]. В этих условиях происходит уменьшение потенциального барьера образца 1 до значения $U_{\max 1}=0.69 \mathrm{eV}$ (табл. 1), удерживающего заряды вблизи твердой поверхности исследуемого объекта. Эффекты перехода структурированной воды в объемное состояние при аналогичных температурах наблюдались также ранее в неорганических и органических средах [9].

Интенсивность термостимулированных токов, исследуемых в температурном интервале от 86 до $89^{\circ} \mathrm{C}$, связана с термическим освобождением слабо закрепленных ионов твердой компоненты и свободных зарядов водной матрицы (рис. 1). В этой области реализуются преимущественно перескоки электретных носителей из одного дефектного узла на другой, а релаксационные процессы имеют, как правило, низкую интенсивность. Релаксация электретных зарядов может быть объясне- 
Таблица 1. Физические параметры электретного состояния образцов мелкодисперсных частиц природного угля разной степени дисперсности $d$ при адсорбции водной компоненты $\varphi=2.1 \%$ в температурном интервале от 27 до $89^{\circ} \mathrm{C}$

\begin{tabular}{c|c|c|c|c|c}
\hline $\begin{array}{c}\text { Дисперсность } \\
d, \mu \mathrm{m}\end{array}$ & $\begin{array}{c}\text { Максимум } \\
\text { термотока }\end{array}$ & $T,{ }^{\circ} \mathrm{C}$ & $I \cdot 10^{-9}, \mathrm{~A}$ & $U_{n}, \mathrm{eV}$ & $\tau, \mathrm{s}$ \\
\hline & 1 & 27 & 10.6 & 0.69 & 166 \\
$31-42$ & 2 & 58 & 8.2 & 0.77 & 184 \\
& 3 & 86 & 6.4 & 0.83 & 199 \\
$54-49$ & 1 & 27 & 8.1 & 0.69 & 166 \\
& 2 & 57 & 6.6 & 0.77 & 183 \\
& 3 & 88 & 4.9 & 0.84 & 200 \\
$82-105$ & 1 & 28 & 7.1 & 0.70 & 167 \\
& 2 & 57 & 5.5 & 0.77 & 183 \\
& 3 & 89 & 2.9 & 0.84 & 201
\end{tabular}

на наличием микроскопических областей в исследуемых структурах, в которых поляризация осуществляется на локальных заряженных центрах [10]. Обнаруженный пик при $86^{\circ} \mathrm{C}$ образца 1 соответствует значению термотока $I_{\max 1}=6.4 \cdot 10^{-9} \mathrm{~A}$ и незначительно превышает максимумы термотоков двух других образцов $I_{\max 2}=4.9 \cdot 10^{-9} \mathrm{~A}$ и $I_{\max 3}=2.9 \cdot 10^{-9} \mathrm{~A}$ (рис. 1 , табл. 1).

Таким образом, экспериментальные результаты свидетельствуют о том, что низкотемпературные максимумы термотоков характеризуют накопление межфазных электретных зарядов под действием внутреннего электрического поля и связаны с особенностями структуры водной компоненты.

Высокотемпературный интервал от 120 до $620^{\circ} \mathrm{C}$ для всех исследуемых объектов, по всей вероятности, связан с разрушением структуры твердой компоненты. Появление высокотемпературных максимумов связано с освобождением электретных зарядов из дефектных областей, расположенных на поверхности и в объеме твердой компоненты частиц природных углей. Выяснено, что в отличии от низкотемпературного участка термостимулированные токи отличаются существенно большей амплитудой и расположены в отрицательной области токов, что свидетельствует, по всей вероятности, об изменении направления напряженности собственного внутреннего электрического поля (рис. 1).

С ростом температуры ионизация структурных дефектов твердой компоненты и, как следствие, концентрация свободных электретных зарядов заметно увеличивается. В таких условиях создается возможность изменения не только модуля внутреннего электрического поля, но и его направления. Обнаружено, что в отличии от низкотемпературных термомаксимумов тока высокотемпературные проявляют зависимость от поверхностной активности исследуемых мелкодисперсных структур (рис. 1).

Значительная амплитуда термотока более активного образца 1 соответствует току $I_{\max 1}=5.0 \cdot 10^{-9} \mathrm{~A}$ при температуре $T_{\max 1}=320^{\circ} \mathrm{C}$, а при увеличении температуры до $471^{\circ} \mathrm{C}$ интенсивность максимумов термотоков этого образца резко увеличивается до значения тока $I_{\max 1}=13.8 \cdot 10^{-9} \mathrm{~A}$ (рис. 2, табл. 2). Увеличение максимума термотоков свидетельствует о возрастании концентрации свободных электретных зарядов на межфазных границах системы в результате термической ионизации структурных дефектов твердокристаллической фазы. Образованные свободные электретные заряды попадают в каналы ионной проводимости и под действием градиентов потенциала внутреннего электрического поля аккумулируют ток. Динамика релаксации этого тока является отражением структуры изучаемой системы.

Экспериментально обнаружено, что при высоких температурах наличие большого количества свободных зарядов, каналов ионной проводимости и внутренней разности потенциалов способствуют возникновению интенсивного переноса электретных зарядов.

Динамика термостимулированных токов позволила выявить, что с уменьшением поверхностной активности твердой компоненты положение пика термостимулированного тока уходит в область высоких температур.

Этот факт можно интерпретировать следующим образом. Энергия активации заряженных локальных дефектов, находящихся на активной поверхности и в объеме механоактивированных мелкодисперсных зерен природных углей, является результирующей функцией вкладов энергии внутреннего электрического и теплового полей. Можно полагать, что электрическая внутренняя энергия, создаваемая более активной поверхностью мелких зерен природных углей образца 1, вносит больший вклад в энергию активации локальных дефектов в сравнении с менее активными образцами 2 и 3. И, как следствие, для более активного образца 1 облегченная внутренним электрическим полем, ионизация заряженных локальных комплексов осуществляется при более низкой температуре. Действительно, в высокотемпературной области максимумы термотока более активного образца 1 проявляются при более низких температурах $T_{\max 1}=320$ и $471^{\circ} \mathrm{C}$ (рис. $\left.1, a\right)$ в сравнении с образцами 2 и 3. Для менее активных экспериментальных образцов 2 и 3 превалирующий вклад в энергию активации вносит тепловое поле, и в связи с этим максимальные значения

Таблица 2. Физические параметры электретного состояния образцов мелкодисперсных частиц природного угля в высокотемпературном интервале от 320 до $620^{\circ} \mathrm{C}$

\begin{tabular}{c|c|c|c|c|c}
\hline $\begin{array}{c}\text { Дисперсность } \\
d, \mu \mathrm{m}\end{array}$ & $\begin{array}{c}\text { Максимум } \\
\text { термотока }\end{array}$ & $T,{ }^{\circ} \mathrm{C}$ & $I \cdot 10^{-9}, \mathrm{~A}$ & $U_{n}, \mathrm{eV}$ & $\tau, \mathrm{s}$ \\
\hline \multirow{3}{*}{$31-42$} & 4 & 320 & 5.0 & 1.38 & 329 \\
& 5 & 471 & 13.8 & 1.73 & 413 \\
$54-69$ & 4 & 328 & 4.5 & 1.40 & 338 \\
& 5 & 490 & 8.3 & 1.78 & 423 \\
$82-105$ & 4 & 335 & 4.3 & 1.41 & 337 \\
& 5 & 520 & 7.4 & 1.85 & 440
\end{tabular}



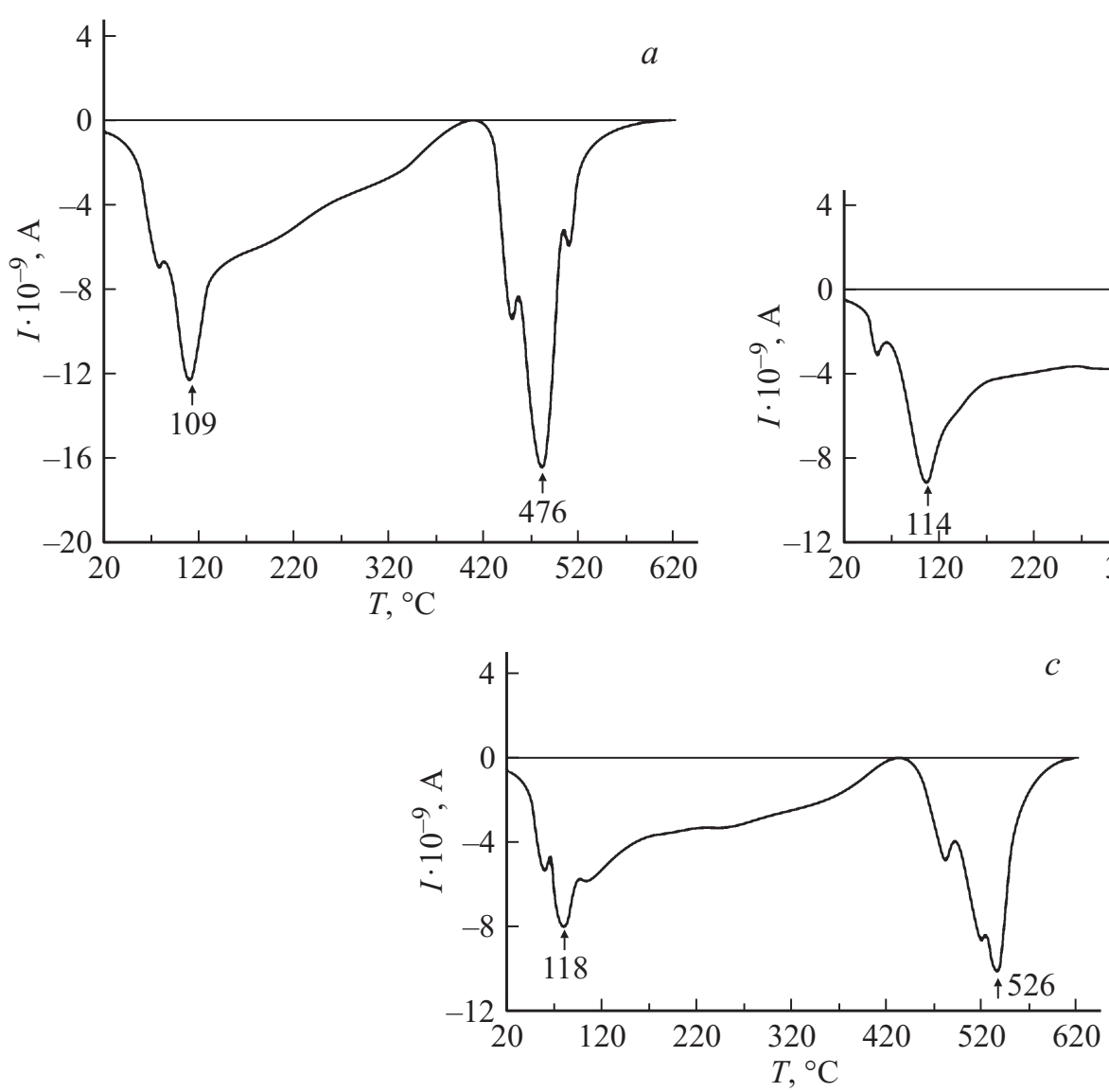

$b$

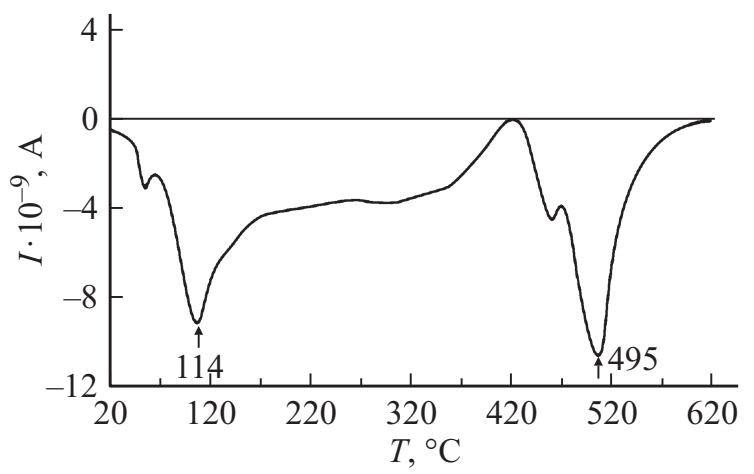

Рис. 2. Спектры термостимулированных токов механоактивированных мелкодисперсных образцов природных углей различной дисперсности и одинаковой величиной адсорбции водной компоненты $\varphi=16.5 \%$ : $a-$ спектры образца 1 (дисперсность 32-42 $\mu \mathrm{m}) ; b-$ спектры образца 2 (дисперсность 54-69 $\mu \mathrm{m}$ ); $c$ - спектры образца 3 (дисперсность 82-105 $\mu \mathrm{m}$ ).

термопиков этих образцов формируются при более высоких температурах $T_{\max 2}=328$ и $490^{\circ} \mathrm{C}$ и $T_{\max 3}=335$ и $520^{\circ} \mathrm{C}$ (рис. $1, b$ и $c$ ) [11].

В высокотемпературной области от 320 до $600^{\circ} \mathrm{C}$ носители электретных зарядов образуются в результате термической ионизации структурных дефектов твердой фазы.

На рис. 2 представлены термограммы исследуемых трех образцов мелкодисперсных частиц минеральных углей с одинаковой величиной адсорбции водной компоненты $\varphi=16.5 \%$ и различной дисперсностью: 31-42 $\mu \mathrm{m}(a), 54-69 \mu \mathrm{m}(b)$ и $82-105 \mu \mathrm{m}(c)$.

При увлажнении поверхность кристаллических частиц минеральных углей частично нарушается. В этих условиях возрастает число слабосвязанных заряженных дефектов, покидающих частицы твердой фазы и внедряющихся в водную компоненту, а также обеспечивается транспорт значительно большей части термически освобожденных зарядов, что и приводит к росту ТСТ (рис. 2).

Экспериментально установлено, что в релаксацию свободных электретных зарядов исследуемых структур вносят значительный вклад адсорбционные процессы, способные изменять спектр поверхностного состояния изучаемых образцов и их диэлектрические свойства.
В исследованном температурном интервале от 20 до $620^{\circ} \mathrm{C}$ при нагревании изучаемых увлажненных объектов в спектрах ТСТ для каждого изучаемого образца обнаружено только два доминирующих термомаксимума, расположенных в отрицательной области (рис. 2). Бо́льшая амплитуда термотока связана с увеличением концентрации водной фазы и свободных электретных зарядов при термическом разрушении твердой компоненты исследуемых объектов. Проявление максимумов тока связано с термическим высвобождением электретных зарядов из дефектных областей, расположенных на поверхности и в объеме частиц минеральных углей (рис. 2) [12].

Анализ экспериментальных исследований показал, что амплитуда термотоков всех исследуемых увлажненных образцов характеризуется значительно возросшей концентрацией свободных зарядов на межфазных границах исследуемых структур.

Электрически более активный образец 1 в исследуемом температурном интервале характеризуется двумя наиболее интенсивными термомаксимумами тока. При температуре $T_{\max 1}=109^{\circ} \mathrm{C}$ первый максимум тока увлажненного образца 1 соответствует максимуму термотока $I_{\max 1}=12.4 \cdot 10^{-9} \mathrm{~A}$, а при более высокой 
Таблица 3. Физические параметры электретного состояния образцов мелкодисперсных частиц природного угля разной дисперсности $d$ и фиксированной величины адсорбции водной компоненты $\varphi=16.5 \%$ в температурном интервале от $100^{\circ} \mathrm{C}$ до $620^{\circ} \mathrm{C}$

\begin{tabular}{c|c|c|c|c|c}
\hline $\begin{array}{c}\text { Дисперсность } \\
d, \mu \mathrm{m}\end{array}$ & $\begin{array}{c}\text { Максимум } \\
\text { термотока }\end{array}$ & $T,{ }^{\circ} \mathrm{C}$ & $I \cdot 10^{-9}, \mathrm{~A}$ & $U_{n}, \mathrm{eV}$ & $\tau, \mathrm{s}$ \\
\hline & 1 & 109 & 12.4 & 0.89 & 212 \\
$31-42$ & 2 & 476 & 16.2 & 1.74 & 417 \\
& 1 & 114 & 8.9 & 0.90 & 215 \\
$54-69$ & 2 & 495 & 10.3 & 1.78 & 426 \\
& 1 & 118 & 8.1 & 0.91 & 217 \\
$82-105$ & 2 & 526 & 10.1 & 1.86 & 444
\end{tabular}

температуре $T_{\max 2}=476^{\circ} \mathrm{C}$ проявляется второй максимум тока этого образца с большей амплитудой тока $I_{\max 2}=16.2 \cdot 10^{-9} \mathrm{~A}$ (рис. 2). В высокотемпературной области происходит интенсивное ослабление связей твердой компоненты, что сопровождается увеличением энергии активации этого образца от $U_{\max 1}=0.89 \mathrm{eV}$ до $U_{\max 2}=1.74 \mathrm{eV}$ (табл. 3). Амплитуда тока двух других менее активных образцов 2 и 3 в этом температурном интервале уступает амплитуде образца 1, что связано с уменьшением концентрации свободных зарядов на межфазных границах исследуемых образцов.

Обнаружено смещение температурного положения максимумов ТСТ в область более высоких температур для менее активных образцов 2 и 3 (рис. 2), что, вероятнее всего, связано с интенсивным экранированием зарядов $\left(\varphi_{3}=16.5 \%\right)$, расположенных на активной поверхности частиц твердой фазы, способствующее уменьшению напряженности внутреннего собственного поля рассматриваемых структур. В связи с этим превалирующий вклад в энергию активации заряженных дефектов вносит тепловое поле и в таких условиях температура формирования термомаксимумов повышается (рис. 2, $b$ и $c$ ).

Результаты электретно-термического анализа, приведенного в настоящей работе, свидетельствуют о том, что в высокотемпературной области интенсивность межфазного взаимодействия, температурное положение максимумов термотока и напряженность собственного внутреннего электрического поля изучаемых гетерогенных систем зависят от величины удельной поверхности электрически активных частиц твердой фазы и концентрации водных пленок [13].

\section{Заключение}

Полученные экспериментальные результаты позволили построить модель процессов, происходящих в исследуемых разупорядоченных мелкодисперсных системах. Основополагающим фактором протекающих процессов на межфазных границах являются интенсивные электри- ческие контакты поверхностей разнородных компонент, осуществляющиеся за счет активных межсоединений, способствующих формированию градиентов потенциала собственного электрического поля и обеспечивающих генерацию внутренней электрической энергии в отсутствие внешнего источника тока. Экспериментально зарегистрированная аккумуляция электрических токов в такой двуфазной неравновесной системе при отсутствии внешнего напряжения является подтверждением формирования градиентов потенциала внутреннего электрического поля в изучаемых структурах.

Вблизи твердой электрически активной поверхности напряженность внутреннего поля максимальна, и молекулы полярной жидкости жестко ориентируются под действием этого поля, образуя наиболее упорядоченный слой молекул. Этот слой представляет собой матрицу для последующих молекулярных слоев и в связи с этим инициирует образование кластеризованных структур в жидкой фазе. Упорядочение молекул жидкой матрицы в объеме этого слоя приводит к уменьшению числа степеней свободы их движения и обусловливает вырождение дипольно-ориентационной поляризации жидкой фазы.

С увеличением расстояния от активной поверхности мелкоразмерных частиц природных углей потенциал и напряженность внутреннего поля уменьшаются и на некотором расстоянии от поверхности твердой компоненты становятся равными нулю. В этих условиях молекулы жидкой полярной воды становятся свободными от действия внутреннего электрического поля и находятся только под действием теплового движения.

Анализ проведенных результатов позволил выяснить, что межфазный слой структурированной связанной воды приобретает функции потенциального барьера как для свободных молекул, так и для молекул, ориентированных электрическим полем активной поверхности мелкодисперсных частиц природных углей. Поскольку молекулы структурированного слоя полярной воды ориентированы собственным внутренним полем и жестко им удерживаются, то их переход в область, где находятся свободные молекулы воды, практически невозможен и является запрещенным переходом.

Для свободных молекул воды в процессе теплового движения возможен случайный переход в область структурированного слоя полярной пленки воды. Однако в этом случае они будут ориентированы внутренним электрическим полем системы и объединены с молекулами структурированного водного слоя. Кроме того, следует учесть, что плотная упаковка молекул в упорядоченном слое водной компоненты служит препятствием к включению в ее структуру дополнительных молекул. Это обстоятельство является также запрещающим фактором проникновения свободных молекул жидкости в область структурированного слоя. Можно заключить, что межфазная область является прочным структурированным слоем воды в мелкоразмерных электрически активных системах. 


\section{Список литературы}

[1] Mascarenhas S. // Ann. N Y. Acad. Sci. 1974. Vol. 238. P. 3652.

[2] Гороховатский Ю.А., Бордовский Г.А. Термоактивационная токовая спектроскопия высокоомных полупроводников и диэлектриков. М.: Наука, 1991. 244 с.

[3] Тареев Б.М. Физика диэлектрических материалов. М.: Энергоиздат, 1982. $320 \mathrm{c}$.

[4] Groenink J.A., Binsma H. // J. Sol. Stat. Chem. 1979. Vol. 29. N 2. P. 227.

[5] Электреты: сборник / Под ред. Г. Сесслера, пер. с англ. А.Ю. Гросберга, Ю.К. Джикаева, пер. под ред. А.Н. Губкина. М.: Мир, 1983. 486 с.

[6] Пат. РФ № 2393476 / Л.А. Щербаченко, Н.Т. Максимова, Л.И. Ружников. Источник электродвижущей силы. БИ № 36. 27. 12. 2010.

[7] Пинчук Л.С., Кравцов А.Г., Зотов С.В. // ЖТФ. 2001. Т. 71. Вып. 5. С. 115-118.

[8] Щербаченко Л.А., Максимова Н.Т., Комаров Е.С. и др. // ЖТФ. 2012. Т. 48. Вып. 10. С. 92-103.

[9] Frenkel Ya.I. // J. Phys. Rev. 1938. Vol. 54. P. 657.

[10] Щербаченко Л.А., Борисов В.С., Максимова Н.Т. и др. // ЖТФ. 2010. Т. 80. Вып. 8. С. 136-142.

[11] Щербаченко Л.А., Максимова Н.Т., Барышников С.С. и др. // ФТТ. 2011. Т. 53. Вып. 7. С. 1417-1422.

[12] Щербаченко Л.А., Максимова Н.Т., Барышников Е.С. и др. // ЖТФ. 2010. Т. 80. Вып. 8. С. 136-142.

[13] Щербаченко Л.А., Борисов В.С. // ФТТ. 2009. Т. 51. Вып. 12. С. 2394-2399. 